\title{
Sidelobe Suppression in Array-Pattern Synthesis Using Periodic Leaky-Wave Antenna and Binomial Array
}

\author{
Hicham Aziz $\mathbb{D}^{\mathbb{D}}$, Mahmoud Moubadir, Abdelkrim Farkhsi, and Naima Amar Touhami \\ Laboratory of Information Systems and Telecommunication, Faculty of Sciences, Abdelmalek Essaadi University, P.O. Box 2121, \\ Tetouan, Morocco \\ Correspondence should be addressed to Hicham Aziz; aziz.hich1am@gmail.com
}

Received 7 October 2019; Revised 5 October 2020; Accepted 14 October 2020; Published 11 November 2020

Academic Editor: Michele Calì

Copyright (C) 2020 Hicham Aziz et al. This is an open access article distributed under the Creative Commons Attribution License, which permits unrestricted use, distribution, and reproduction in any medium, provided the original work is properly cited.

\begin{abstract}
This paper presents the conception and realization of a 2D antenna array using periodic leaky-wave antenna (PLWA) and the binomial array (BA) at $6 \mathrm{GHz}$ as the application of WLAN. The series array of periodic leaky-wave antenna was provided by an array of five rectangular Patches connected by cross lines. The nonuniform amplitudes of the binomial array are used to reduce the sidelobe level; in this way, the center source radiates strongly on the broadside. The prototype of the proposed $2 \mathrm{D}$ antenna array is designed, fabricated, and tested. A good agreement is obtained between simulated and measurement results.
\end{abstract}

\section{Introduction}

Nowadays, the practical applications of microwave antennas pose problems in performances, especially at the sidelobe level (SLL). In most applications of antenna arrays, sidelobe levels are commonly unwanted, especially the first sidelobe which is the main source of electromagnetic interference. This later should be reduced without deforming the main beam of the radiation pattern.

There are several methods to reduce the sidelobe level such as Minmax [1], Dolph-Chebyshev [2], genetic algorithm [3], Particle Swarm Optimization (PSO) [4], and binomial method $[2,5]$. We used the binomial method because it gives a good performance with a minimum sidelobe level than the others [6]. Other methods such as in [7-9], using the Real Coded Genetic Algorithm (RCGA), result in a larger array size than the original, which may be considered a drawback. To reduce the sidelobe level of the array antenna, we must take into account scan direction, beam width, and type of antenna element used.

The binomial array (BA) is a nonuniform amplitude distribution with element spacing equal (less than $\lambda$ ) to possess the smallest sidelobes $[5,10]$. It is obvious that the designer has to make a compromise between the level of sidelobes and the width of the beam. The amplitude distribution along the source is a criterion that can be used to evaluate the sidelobe level and the relative beam width.

The leaky wave is a fast wave that has been extensively studied and used for more than four decades [11, 12], while the first planar leaky-wave antennas were reported in the 1940s [13, 14], mainly planar periodic leaky-wave antennas, due to having various advantages, such as low cost, simple fabrication, lightweight, and integration with electronic components. These are often found in several microwave applications, for example, communication systems such as satellite links and sensor systems [15]. An overview of recent advances in this area is available [16].

The periodic leaky-wave antenna is characterized by high directivity and wide beam width. Furthermore, their radiation direction and main beam angle may be steered by varying the excitation frequency or by using electronically tunable circuit elements [17].

In order to minimize the sidelobe level, in this paper, we combine the periodic leaky-wave antenna and the binomial 


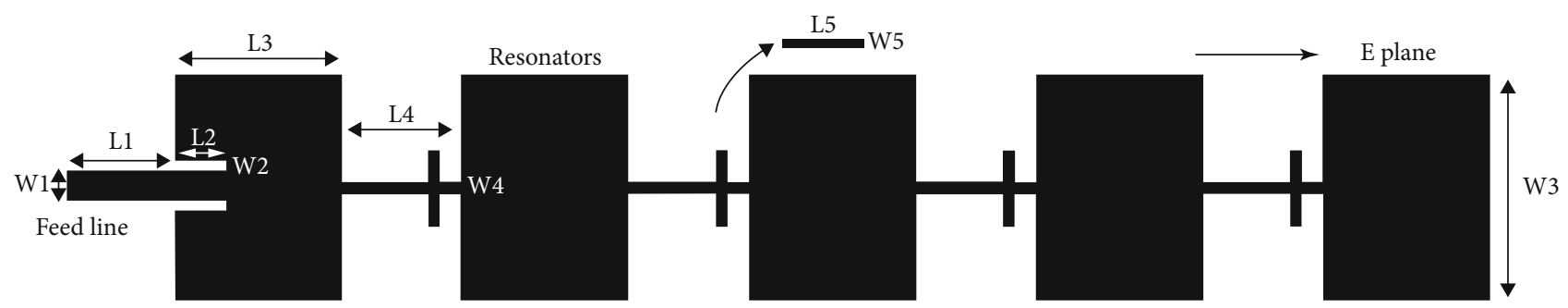

FIGURE 1: Series of the periodic leaky-wave antenna.

TABLE 1: Length and width of the leaky-wave structure.

\begin{tabular}{lccc}
\hline Parameter & Value $(\mathrm{mm})$ & Parameter & Value $(\mathrm{mm})$ \\
\hline L1 & 2.85 & W1 & 7.51 \\
L2 & 3.72 & W2 & 0.53 \\
L3 & 11.5 & W3 & 15.35 \\
L4 & 7.5 & W4 & 0.4 \\
L5 & 6 & W5 & 0.4 \\
\hline
\end{tabular}

method to design a $2 \mathrm{D}$ antenna array. The high directivity and wide beam width of a periodic leaky-wave antenna are used to characterize the series array. To reduce the sidelobe level, the binomial method is used to feed the power network of the parallel array antenna.

\section{Leaky-Wave Antenna}

The leaky-wave antenna is a periodic modulation of the guiding structure. This modulation is introduced along the longitudinal direction of the antenna. In the uniform structures, the fundamental mode is a slow wave $\left(\beta>k_{0}\right)$, which therefore would not radiate even if the structure is electromagnetically open. However, due to the periodic corrugation, the guided wave consists of an infinite number of space harmonics, with longitudinal wave number.

$$
k_{z, n}=k_{z, 0}+\frac{2 \pi n}{p}
$$

where $k_{z, 0}$ is the wave number of the fundamental mode, $n$ is the harmonic order, and $p$ is the period in the longitudinal direction.

One of the main advantages of periodic leaky-wave antennas, compared to uniform structures, is the fact that the phase constant of the leaky mode can assume either positive or negative values at will, which allows scanning the main beam from the backward to the forward quadrant as the frequency is increased. However, the antenna performance generally degrades when we approach broadside, due to the presence of an open stop band of the periodic structure [18]. From a physical viewpoint, at the open stopband frequency, the traveling wave supported by the periodic leaky-wave antenna becomes a standing wave, behaving similarly to an antenna array in which all the radiating elements are excited with the same phase [12]. As a result, the attenuation constant drops to zero and all the reflections at the peri-
TABLE 2: Distribution of currents using the binomial method.

\begin{tabular}{lc}
\hline Series patch antenna & Currents \\
\hline Antenna 1 & 0.16 \\
Antenna 2 & 0.66 \\
Antenna 3 & 1 \\
Antenna 4 & 0.66 \\
Antenna 5 & 0.16 \\
\hline
\end{tabular}

odic discontinuities add in phase back to the input port, determining a purely reactive input impedance and large mismatch.

The proposed Periodic Leaky-wave antenna is shown in Figure 1. The antenna array consists of five rectangular patches interconnected by a high impedance microstrip line. In order to miniaturize the space between the array elements, the cross line is used [19], and there is no theoretical method to calculate it.

The dimensions of our series of the periodic leaky-wave antenna are shown in Table 1.

\section{Binomial Array}

The binomial array was investigated and proposed by J. S. Stone [16] to reduce the sidelobe level; that is, the nonuniform amplitudes of $n$ isotropic sources are arranged according to the coefficient of successive terms of the following:

$$
\begin{aligned}
(1+x)^{n}= & 1+(n-1) x+\frac{(n-1)(n-2) x^{2}}{2 !} \\
& +\frac{(n-1)(n-2)(n-3) x^{3}}{3 !}+\cdots
\end{aligned}
$$

where $n$ is the number of the radiating source in the array and $x$ is a random variable.

The amplitude of each element must have currents proportional to the coefficient of the binomial series to have a reduction of the sidelobe level. In this case, the center source radiates strongly than the broadside.

In this paper, the proposed antenna consists of five elements in parallel with the previous structure in Figure 1 and the separation distance between them is 0.7 $\lambda[20]$. Besides that, we used the binomial method to calculate the impedance of the power network. The currents that feed each series antenna element are similar to those in Table 2. 


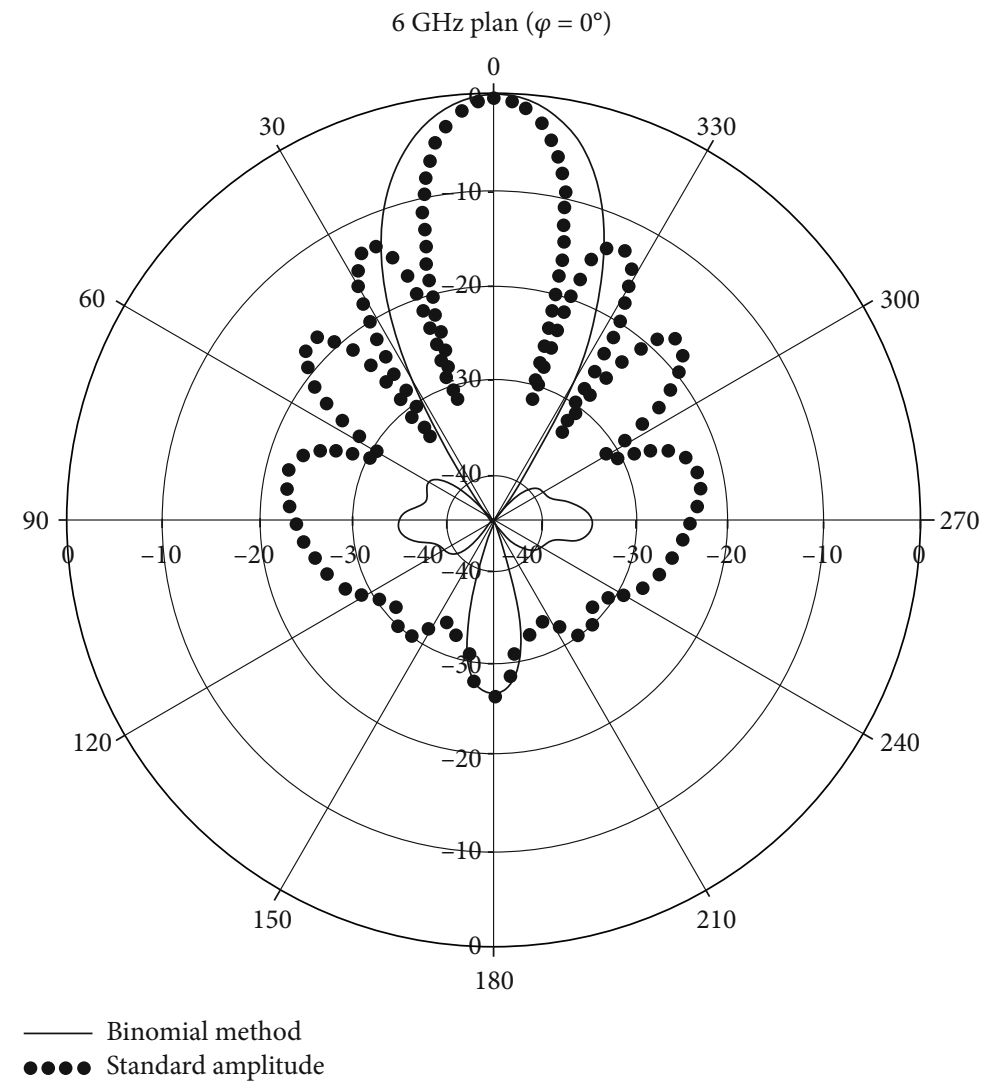

FIgURE 2: Radiation pattern using the binomial method as shown in Table 2 and standard amplitude.

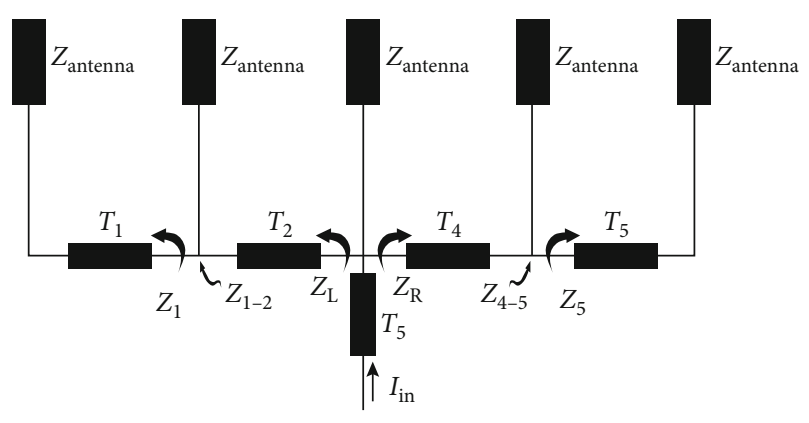

Figure 3: Circuit of impedance diagram.

Figure 2 shows the comparison of the radiation pattern between the binomial and uniform arrays. We can see the high reduction of the sidelobe level using the binomial method.

Figure 3 shows the circuit of a power network used to feed the array antenna. In this power, the antennas were replaced by their impedance equivalent. The $\lambda / 4$ transformers is used to realize the impedance transformation.

The proposed solution was made to obtain the impedance values at each line intersection. The electrical distances from the central cross to each antenna must guarantee that the phase difference between the currents entering each antenna element is approximately equal to zero in order to

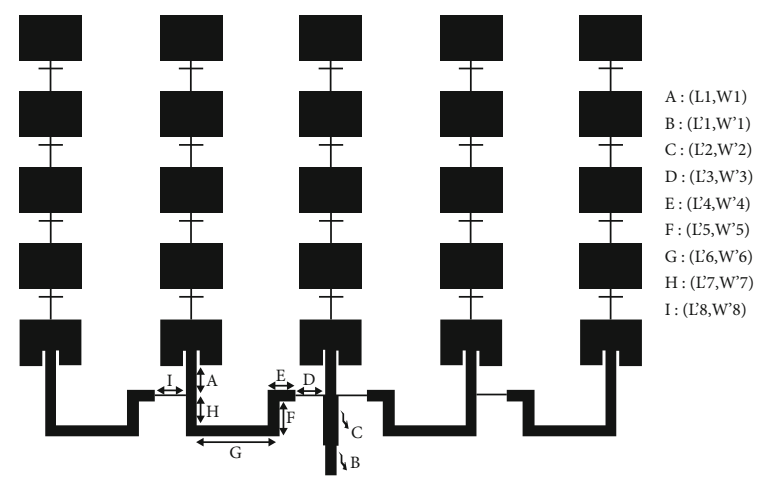

FIGURE 4: 2D antenna array in five elements.

TABLE 3: Length and width of the power network.

\begin{tabular}{lccc}
\hline Parameter & Value $(\mathrm{mm})$ & Parameter & Value $(\mathrm{mm})$ \\
\hline $\mathrm{L} 1$ & 7.5 & $\mathrm{~W} 1$ & 2.85 \\
$\mathrm{~L}^{\prime} 1$ & 7.5 & $\mathrm{~W}^{\prime} 1$ & 2.85 \\
$\mathrm{~L}^{\prime} 2$ & 12.3 & $\mathrm{~W}^{\prime} 2$ & 4 \\
$\mathrm{~L}^{\prime} 3$ & 7 & $\mathrm{~W}^{\prime} 3$ & 0.4 \\
$\mathrm{~L}^{\prime} 4$ & 6.85 & $\mathrm{~W}^{\prime} 4$ & 2.85 \\
$\mathrm{~L}^{\prime} 5$ & 8.85 & $\mathrm{~W}^{\prime} 5$ & 2.85 \\
$\mathrm{~L}^{\prime} 6$ & 23.42 & $\mathrm{~W}^{\prime} 6$ & 2.85 \\
$\mathrm{~L}^{\prime} 7$ & 7.13 & $\mathrm{~W}^{\prime} 7$ & 2.85 \\
$\mathrm{~L}^{\prime} 8$ & 7.57 & $\mathrm{~W}^{\prime} 8$ & 0.6 \\
\hline
\end{tabular}




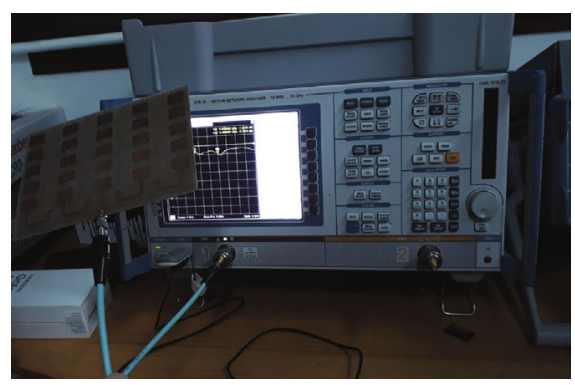

(a)

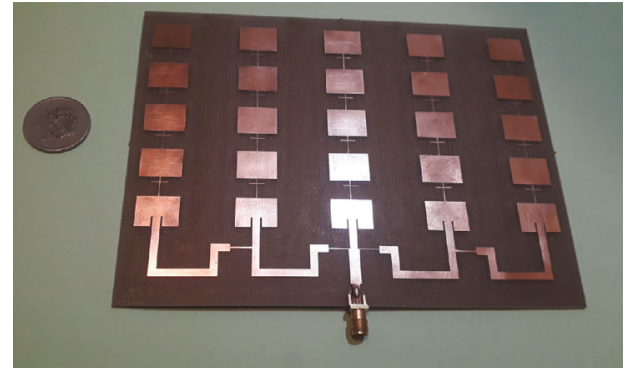

(b)

Figure 5: (a) Measurement was performed with a vector network analyzer (ZVB20). (b) The prototype of the proposed 2D antenna array.

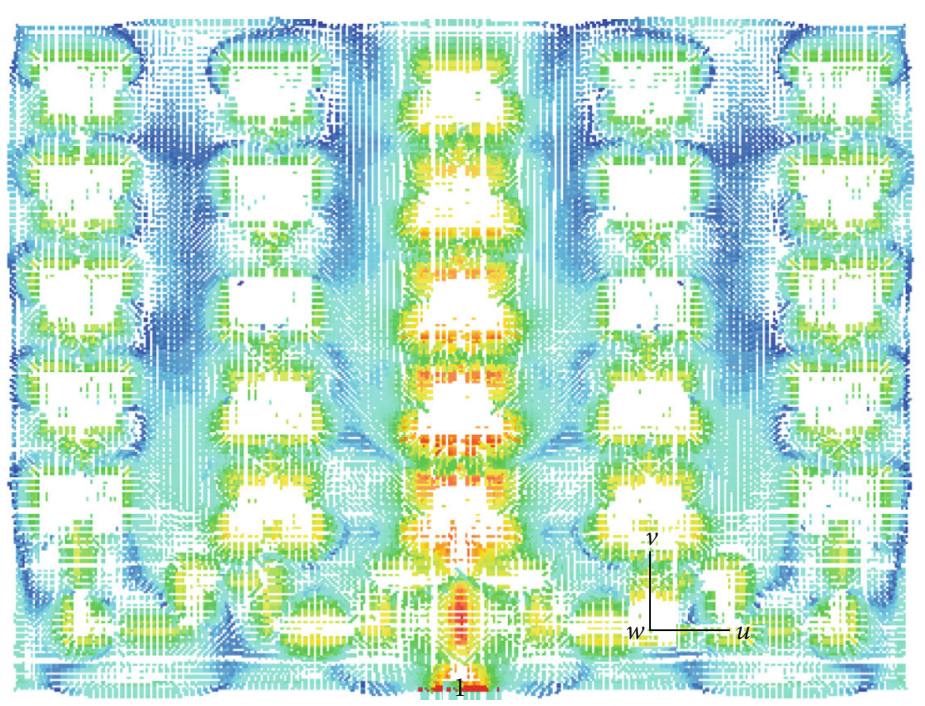

$\mathrm{A} / \mathrm{m}^{2}(\log )$

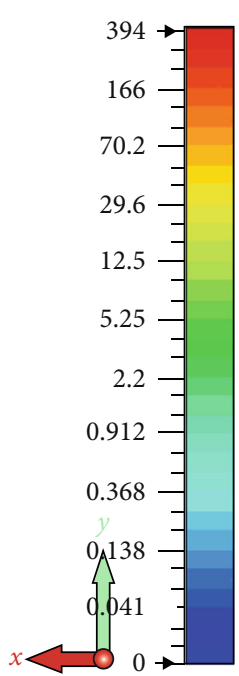

Figure 6: Surface field distribution of the array antenna.

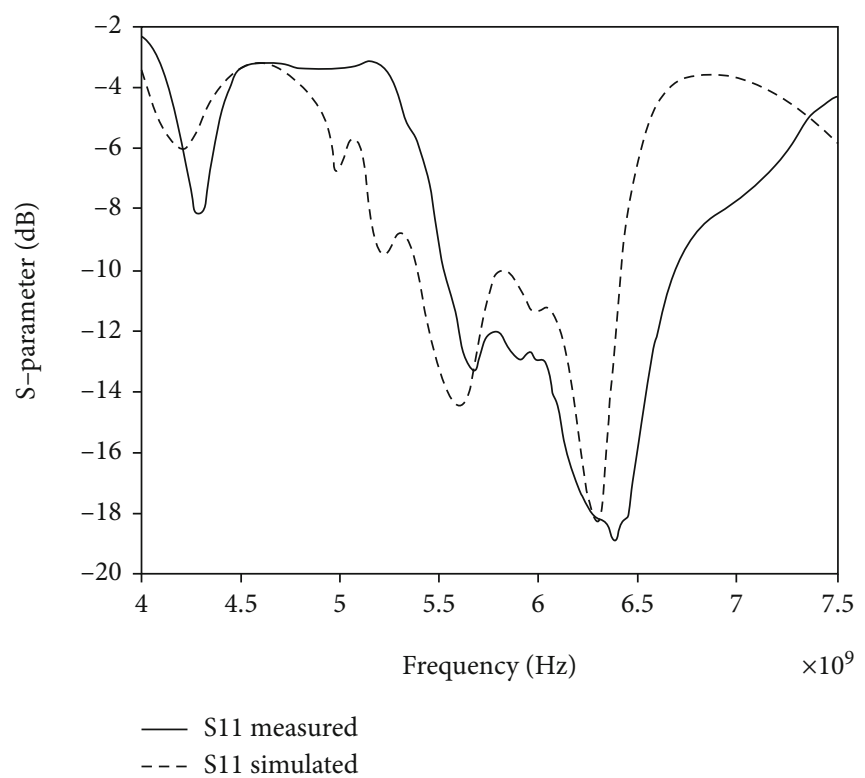

FiguRE 7: Return loss simulated and measured. 


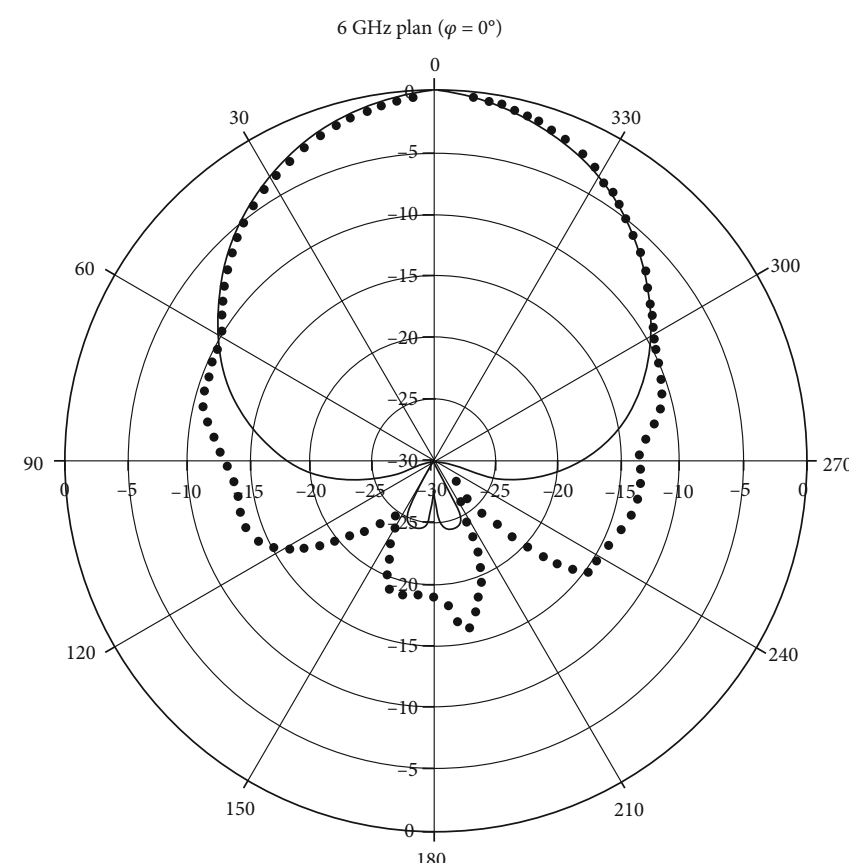

- Simulated

(a)

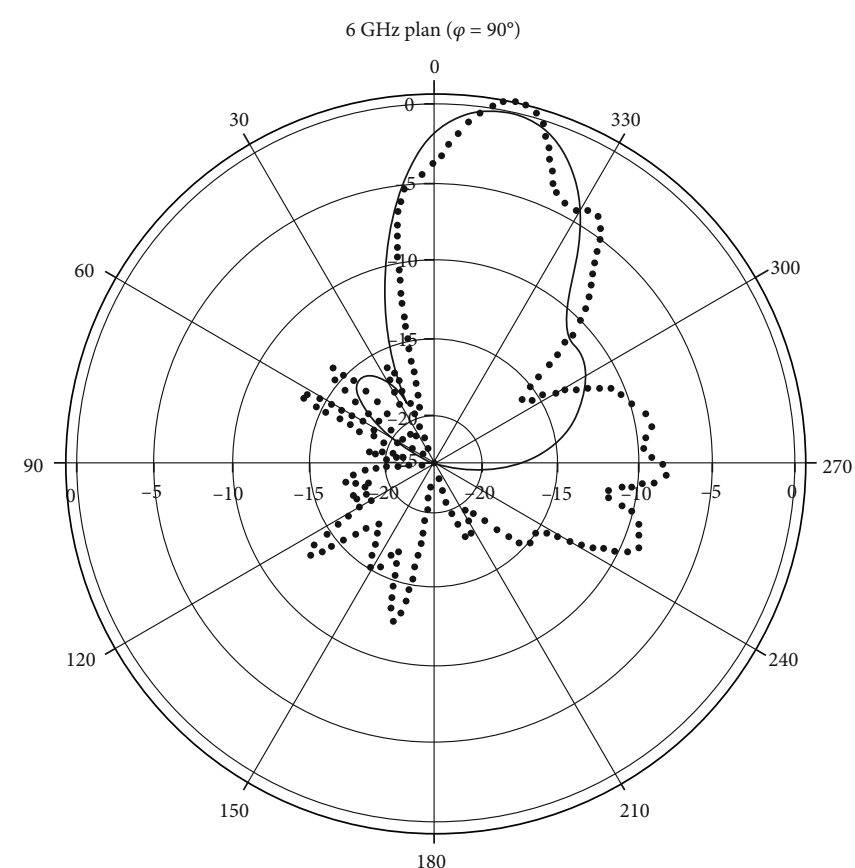

Simulated

-... Measured

(b)

FIGURE 8: (a) Radiation pattern at $6 \mathrm{GHz}$ in the plan $\left(\varphi=0^{\circ}\right)$. (b) Radiation pattern at $6 \mathrm{GHz}$ in the plan $\left(\varphi=90^{\circ}\right)$.

guarantee the vertical pointing. This distance can be obtained through

$$
L=\frac{\lambda}{4}+\left(3-n_{\text {antenna }}\right) \lambda
$$

\section{Results and Discussion}

4.1. Structure. The proposed array antenna is shown in Figure 4. In this design, the substrate FR4 is used due to its low cost and easy fabrication. The substrate height is $1.5 \mathrm{~mm}$, the value of the Dielectric Constant is 4.3 , and the loss tangent is 0.025 . The dimensions of our antenna are optimized by using the electromagnetic software (see Table 3). The array conception is based on our previous research $[19,20]$.

In order to measure the S-parameters of the proposed 2D antenna array, we have been employing the Schwarz ZVB 20 vector network analyzer as shown in Figure 5, in which frequency range is limited to $20 \mathrm{GHz}$.

4.2. Current Distribution. Figure 6 shows that the array antenna has the strongest field at the middle series patch than the broadsides indicating an appropriate implementation of power distribution.

4.3. S-Parameter. Figure 7 shows the simulated and measured return loss (S11) of the proposed antenna array. The simulated and measured return loss of the proposed array is $19 \mathrm{~dB}$ and $18 \mathrm{~dB}$ at a $6 \mathrm{GHz}$ frequency, respectively. The sim- ulation and measurement performances are in good correlation. The measured bandwidth is $1.1 \mathrm{GHz}$.

4.4. Radiation Pattern. Another important issue in the design of the antenna array is the radiation pattern. Figure 8 shows the radiation pattern of our array at $6 \mathrm{GHz}$ frequency in the plan $\left(\varphi=0^{\circ}\right.$ and $\left.\varphi=90^{\circ}\right)$. We can see that the maximum radiation gain is about $10.37 \mathrm{~dB}$ and lower sidelobe level of $24.7 \mathrm{~dB}$ at $6 \mathrm{GHz}$. The measured and simulated results are in correlation, and the antenna radiates in the main beam. It is clear that radiation patterns of the proposed antenna have unidirectional characteristics.

\section{Conclusion}

In this paper, we have presented a $2 \mathrm{D}$ antenna array with a lower sidelobe level. The proposed 2D array is based on a periodic leaky-wave antenna (PLWA) combined with the binomial array (BA). The first one consists of a series of rectangular antennas connected by a cross; the goal of it is to have a reduction in antenna array size and maintain the radiation in the main direction $\left(0^{\circ}\right)$. The second one is to connect 5 elements of (PLWA) by network adaptation to reduce the sidelobe level and have good performances in return loss, radiation pattern, and antenna gain. A good agreement is obtained between simulated and measured results. The proposed antenna is simple and easy to manufacture, which will make it suitable for application in WLAN or radar system. 


\section{Data Availability}

All data generated during this study are included in this published article.

\section{Conflicts of Interest}

The authors declare that they have no conflicts of interest.

\section{Acknowledgments}

The authors would like to thank the Department of Engineering of Communications, University of Cantabria.

\section{References}

[1] F. Harrou, B. Bouyeddou, S. A. Djennas, and B. Merad, "Synthèse et optimisation de réseaux d'antennes. Imprimées linéaires par la méthode du minimax," in 5th International Conference, Sciences of Electronic, Technologies of Information and Telecommunications, Tunisia, March 2009.

[2] J. Franois, D. Essiben, M. P. M. Zanga, E. R. Hedin, and Y. S. Joe, "Design of non-uniform linear antenna arrays using Dolph-Chebyshev and binomial methods," International Journal of Engineering Research and Applications, vol. 5, no. 10, pp. 187-195, 2015.

[3] F. J. Ares-Pena, J. A. Rodriguez-Gonzalez, E. VillanuevaLopez, and S. R. Rengarajan, "Genetic algorithms in the design and optimization of antenna array patterns," IEEE Transactions on Antennas and Propagation, vol. 47, no. 3, pp. 506510, 1999.

[4] T. B. Chen, Y. B. Chen, Y. C. Jiao, and E. S. Zhang, "Synthesis of antenna array using particle swarm optimization," in 2005 Asia-Pacific Microwave Conference Proceedings, p. 4, Suzhou, China, December 2005.

[5] H. Aziz, M. Moubadir, N. Amar Touhami, A. Farkhsi, and A. Tazon, "Reducing side lobes of antenna array by the binomial method," International Journal of Microwave and Optical Technology, vol. 11, no. 1, pp. 40-44, 2016.

[6] M. S. Tavazoei and M. Haeri, "Comparison of the existing methods in determination of the characteristic polynomial," World Academy of Science, Engineering and Technology, vol. 6, pp. 130-133, 2007.

[7] S. Das, S. Bhattacherjee, D. Mandal, and A. K. Bhattacharjee, "Optimal sidelobe reduction of symmetric linear antenna array using genetic algorithm," in 2010 Annual IEEE India Conference (INDICON), pp. 1-4, Kolkata, India, December 2010.

[8] H. Oraizi and M. Fallahpour, "Sum, di erence and shaped beam pattern synthesis by non-uniform spacing and phase control," IEEE Transactions on Antennas and Propagation, vol. 59, no. 12, pp. 4505-4511, 2011.

[9] A. Parsa and H. Oraizi, "Synthesis of planar continuous current sources with arbitrary shapes for generation of desired radiation patterns by methods of moments and least squares," IET Microwaves, Antennas and Propagation, vol. 14, no. 5, pp. 348-359, 2020.

[10] R. L. Yadava, Antenna and Wave Propagation, Eastern Economy Edition, Technical Publications, 2011.

[11] A. Hessel and A. Theory, General Characteristics of TravelingWave Antennas, R. E. Collin and R. F. Zucker, Eds., McGrawhill, New York, 1969.
[12] A. A. Oliner and D. R. Jackson, Antenna Engineering Handbook, J. Volakis, Ed., McGraw-Hill, New York, 4th Ed edition, 2007.

[13] S. Otto, A. Rennings, K. Solbach, and C. Caloz, "Transmission line modeling and asymptotic formulas for periodic leakywave antennas scanning through broadside," IEEE Transactions on Antennas and Propagation, vol. 59, no. 10, pp. 3695-3709, 2011.

[14] P. Aditya, Leaky-Wave Antenna, M.S. thesis, B.E., Visvesvaraya Technological University, 2007.

[15] S. Otto, A. Rennings, K. Solbach, and C. Caloz, "Analysis and optimization of leaky-wave radiation at broadside from a class of 1-D periodic structures," IEEE Transactions on Antennas and Propagation, vol. 54, no. 9, pp. 2593-2604, 2006.

[16] C. Hessel, D. R. Jackson, and T. Itoh, Frontiers in Antennas, F. Gross, Ed., McGraw-Hill, New York, 2010.

[17] S. Paulotto, P. Baccarelli, F. Frezza, and D. R. Jackson, "Fullwave modal dispersion analysis and broadside optimization for a class of microstrip CRLH leaky-wave antennas," IEEE Transactions on Antennas and Propagation, vol. 56, no. 12, pp. 2826-2837, 2008.

[18] S. Paulotto, P. Baccarelli, F. Frezza, and D. R. Jackson, “A novel technique for open-stopband suppression in 1-D periodic printed leaky wave antennas," IEEE Transactions on Antennas and Propagation, vol. 57, no. 7, pp. 1897-1906, 2009.

[19] H. Aziz, M. Moubadir, N. Amar Touhami, and A. Farkhsi, "Planar leaky wave antenna at 6GHz," in 11th international conference Interdisciplinarity in engineering, Tirgu-Mures, Romania, 2017.

[20] H. Aziz, M. Moubadir, N. Amar Touhami, A. Farkhsi, and M. Boussouis, "Influence de variation de la distance de séparation entre les antennes patch," in Congres Mediterraneen des Télécommunications et Exposition, Mohammedia, Marocco, 2014. 